\title{
Nutraceuticals in Neurodegeneration and Aging
}

\author{
Wei-Yi Ong ${ }^{1} \cdot$ Grace Y. Sun ${ }^{2} \cdot$ W. Gibson Wood ${ }^{3} \cdot$ Teng-Nan Lin ${ }^{4}$
}

Published online: 9 August 2016

(c) Springer Science+Business Media New York 2016

Age-related neurodegenerative diseases, such as stroke, vascular dementia, Alzheimer's disease and Parkinson's disease are major health problems worldwide. While great strides have been made toward identifying potential mechanisms for these diseases, effective treatments have remained elusive. There is a growing interest in the use of natural products or herbal medicines for the prevention and treatment of neurodegenerative diseases. The papers of this Special Issue are focused on natural products and neurodegenerative diseases, which resulted from a satellite symposium entitled "Nutraceuticals in Neurodegeneration and Aging" held at the National University of Singapore on August 20-21, 2015. This meeting was carried out in conjunction with the 25th International Society for

Grace Y. Sun

sung@missouri.edu

Wei-Yi Ong

wei_yi_ong@nuhs.edu.sg

W. Gibson Wood

woodx002@umn.edu

Teng-Nan Lin

bmltn@ibms.sinica.edu.tw

1 Department of Anatomy, Yong Loo Lin School of Medicine, National University of Singapore, Singapore 119260, Singapore

2 Department of Biochemistry, University of Missouri, Columbia, MO, USA

3 Department of Pharmacology, University of Minnesota School of Medicine, Geriatric Research, Education and Clinical Center, VA Medical Center, Minneapolis, MN, USA

4 Institute of Biomedical Sciences, Academia Sinica, Taipei, Taiwan, ROC
Neurochemistry (ISN) and the 13th Asian Pacific Society for Neurochemistry (APSN) Biennial Meeting.

This Special Issue contains both reviews and original articles that critically examine different natural products, their mechanisms of action, usage, and potential for development as nutraceuticals in the treatment/prevention of neurodegenerative diseases. The term 'nutraceutical' embodies the age-old concept that regular consumption of these herbal products can be used for the prevention or treatment of diseases. This Issue consists of 20 articles that are divided into four sections: (1) special herbal botanicals including Ashwagandha (Indian Ginseng), Lycium barbarum, Scutellarin, and Clinacanthus nutans (Sabah snake grass), which are known remedies for improving health and treatment of diseases; (2) botanicals that impact on neurological diseases and aging, including ischemic stroke, Parkinson's disease, and Rett syndrome; (3) natural products associated with lipid metabolites and enzymes, e.g., uncommon sphingolipids and phosphatides that bear health effects to the brain; (4) commentaries, new approaches and future directions. Many articles place emphasis on effects of these natural products on reparation of mitochondrial dysfunctions, on protection of damaged neurons and on mitigating inflammatory responses in microglial cells.

These contributions are only a small example of the current interest and research on the use of nutraceuticals in preventing/treating neurodegenerative diseases and agerelated changes in brain function. Indeed, there is a pressing need for further studies to better understand the mechanisms of action of nutraceuticals, and development of new candidates that are efficacious. This Special Issue will appeal to not only to neuroscientists but also to those interested in phytochemistry and brain health.

Photos of some of the speakers and participants are enclosed. 

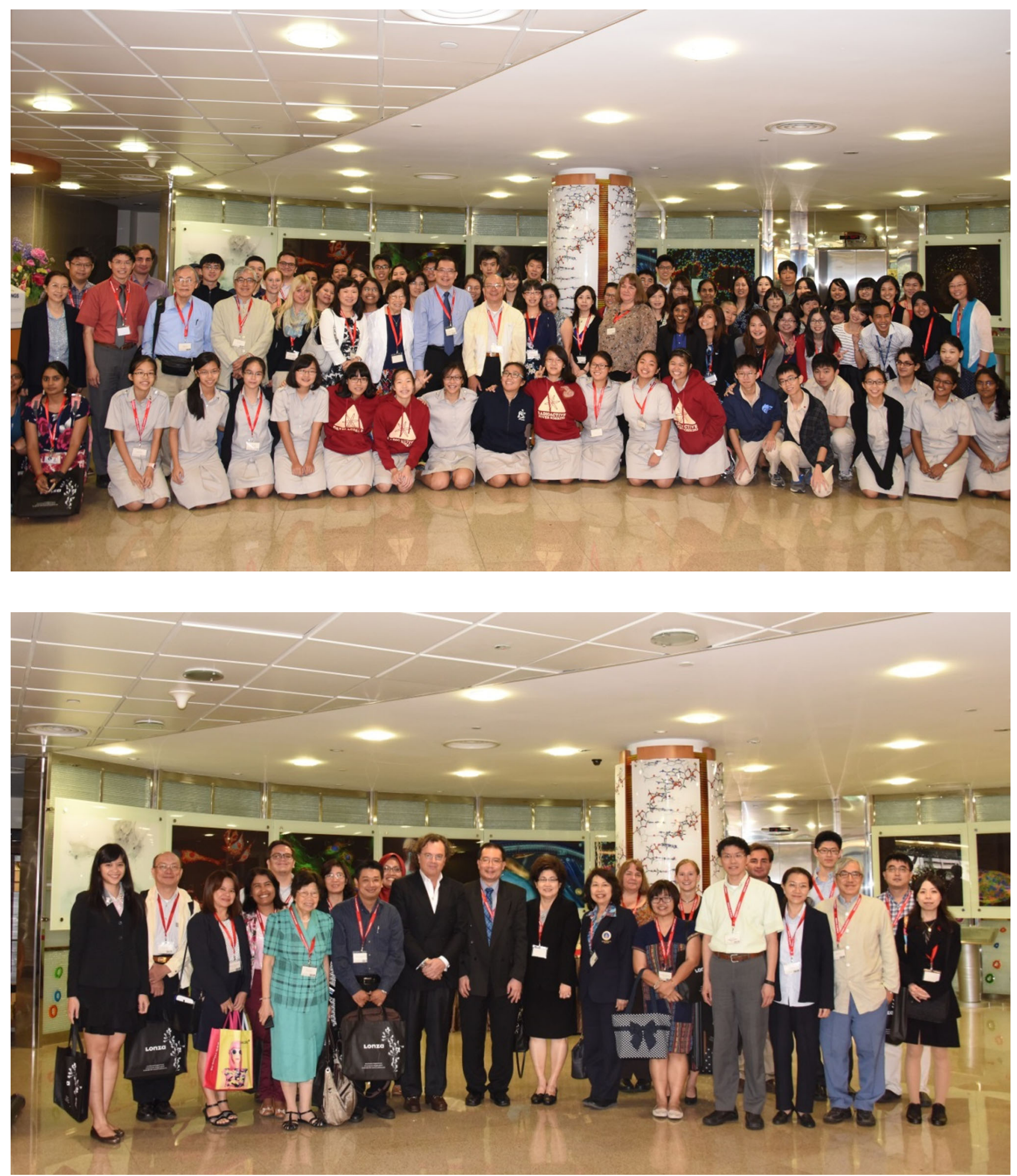